\title{
Erratum to: MIR closures of polyhedral sets
}

\author{
Sanjeeb Dash • Oktay Günlük • Andrea Lodi
}

Received: 13 October 2009 / Accepted: 13 October 2009 / Published online: 19 November 2009

(C) Springer and Mathematical Programming Society 2009

\section{Erratum to: Math. Program., Ser. A (2010) 121:33-60 DOI 10.1007/s10107-008-0225-x}

In Section 3.3 of the paper [3], we compare our nonlinear separation model (MIR-SEP) for MIR cuts with the one for split cuts (PMILP) presented in Balas and Saxena [1] and show that (Lemma 9) they are equivalent. We then present a numerical example to show that the linearized separation models are not equivalent and make the following claim without a proof:

"The Balas/Saxena model PMILP for this example (or more precisely, the deparametrized model MILP( $\theta)$ ) is infeasible unless the parameter $\theta$ is chosen to be exactly $0.31 . "$

This claim is incorrect (as pointed out to us by Balas and Saxena) and contains two errors. The first is that Balas and Saxena [1] assume that all variables are non-negative, whereas the example in our paper contains a free variable. Secondly, for any problem in the correct format (including the one obtained by replacing the free variable in our example by two non-negative variables) $\operatorname{MILP}(\theta)$ is feasible for all $\theta \in(0,1)$.

The online version of the original article can be found under doi:10.1007/s10107-008-0225-x.

S. Dash $(\varangle) \cdot$ O. Günlük

IBM, T.J. Watson Research Center, P.O. Box 218, Yorktown Heights, NY 10598, USA

e-mail: sanjeebd@us.ibm.com

O. Günlük

e-mail: gunluk@us.ibm.com

A. Lodi

DEIS, University of Bologna, viale Risorgimento 2, 40136 Bologna, Italy

e-mail: andrea.lodi@unibo.it 
However, it is still true that the linearized separation models are not equivalent. More precisely, it is possible to show the following claim. For the set $Q=\left\{x_{1} \in \mathbb{R}, x_{2}, x_{3} \in\right.$ $\left.\mathbb{Z}: x_{1}+x_{2}-x_{3} \geq 0.31, x_{1}, x_{2}, x_{3} \geq 0\right\}$, the separation model MILP $(\theta)$ in [1] cannot produce the single cut that defines the MIR closure of $Q$ unless $\theta$ is chosen to be one of 0.31 or $1-0.31=0.69$. In other words, the MIR cut $x_{1}+0.31\left(x_{2}-x_{3}\right) \geq 0.31$ is not a feasible solution to $\operatorname{MILP}(\theta)$ if $\theta \notin\{0.31,1-0.31\}$. This claim remains true if 0.31 is replaced by any number in $(0,1)$. Therefore, the solution set of Appx-MIRSep with, say, $k=2$ is not contained in the union of the solution sets of MILP(0), MILP(1/4), and MILP(1/2).

Note that Balas and Saxena do not use the cut generated by $\operatorname{MILP}(\theta)$ directly, but instead use the disjunction obtained by $\operatorname{MILP}(\theta)$ to generate a second cut via a linear program that uses a special normalization constraint. For the example above, the cut generated by this second LP is then strengthened [2] using the Balas-Jeroslow monoidal strengthening technique to obtain the desired MIR cut.

\section{References}

1. Balas, E., Saxena, A.: Optimizing over the split closure. Math. Program. Ser. A 113, 219-240 (2008)

2. Balas, E.: personal communication (2009)

3. Dash, S., Günlük, O., Lodi, A.: On the MIR closure of polyhedral sets. Math. Program. Ser. A 121, 3360 (2010) 